\title{
VARIACIÓN TEMPORAL EN LA ESTRUCTURA DE LOS RECURSOS EN UN SITIO DEL CHACO HÚMEDO ARGENTINO
}

\section{TEMPORAL VARIATION IN THE RESOURCES STRUCTURE AT ARGENTINE HUMID CHACO REGION SITE}

\author{
María Macarena Zarza1, Luis Manuel del Papa² y Guillermo Nicolás Lamenza ${ }^{3}$ \\ ${ }^{1}$ División Antropología, Facultad de Ciencias Naturales y Museo, UNLP. \\ E-mail: macarena_zarza@hotmail.com \\ ${ }^{2}$ Cátedra de Anatomía Comparada, Facultad de Ciencias Naturales y Museo, UNLP; CONICET. \\ E-mail: loesdelpapa@hotmail.com \\ ${ }^{3}$ División Antropología, Facultad de Ciencias Naturales y Museo, UNLP. CONICET. \\ E-mail: guillermolamenza@gmail.com
}

Presentado: 04/04/2019 - Aceptado: 20/09/2019

\section{Resumen}

Las primeras investigaciones zooarqueológicas en la región chaqueña permitieron esclarecer los principales aspectos de la subsistencia humana durante el Holoceno tardío. En estos aportes pioneros, los restos faunísticos se analizaron teniendo en cuenta a cada sitio arqueológico como representante de una sola ocupación. Sin embargo, un posterior análisis de los conjuntos cerámicos siguiendo técnicas numéricas multivariadas propuso la existencia de múltiples ocupaciones en algunos de los sitios. Con esta información se seleccionaron dos agrupamientos de un sitio en particular (El Cachapé Potrero IVB), y se realizaron fechados radiocarbónicos

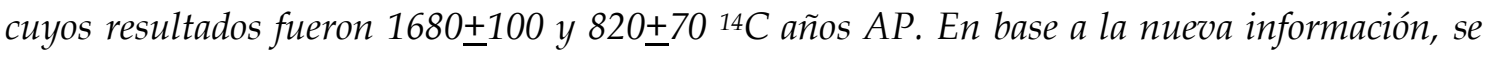
decidió realizar la revisión de la arqueofauna, con el objetivo de corroborar la existencia de variación temporal en la estructura de los recursos. Para ello se efectuó la determinación anatómica y taxonómica de los restos, el análisis tafonómico y la cuantificación de la muestra en forma de NISP, \%NISP y MNI, e indices de Riqueza, Diversidad, Equitatividad y abundancia relativa. Estos resultados permitieron generar nueva información de utilidad para complementar y ajustar el modelo de explotación de recursos, en relación con la variación a lo largo del tiempo de las ocupaciones humanas ribereñas chaqueñas.

Palabras clave: Holoceno tardío, componentes arqueológicos, sistema de subsistencia ribereño.

\section{Abstract}

The first zooarchaeological investigations in the region of Chaco allow to clarify the main aspects of human subsistence during the late Holocene. In these pioneering contributions, the faunal remains were analyzed considering each archaeological site as representative of a single 
occupation. However, a subsequent analysis of pottery assemblages following numeric multivariate techniques, proposed the existence of more than one occupation in some of the sites. With this information, two clusterwere selected in a particular site (El Cachapé Potrero IVB), radiocarbon dating were made and the results were $1680 \pm 100$ and $820 \pm 70{ }^{14} \mathrm{C}$ years BP. Based on the new information, it was decided to review the archaeofauna remains in order to corroborate the existence of temporal variation in the resources structure. For this, we perform the anatomical and taxonomic determination of the remains, the taphonomic analysis and the quantification of the taxonomic abundance of the sample in the form of NISP, \% NISP and MNI, and Richness, Diversity, Equitativity and relative abundance index. These results allow us to generate new useful information to complement and adjust the model of resources exploitation in order to the variation over time of the human occupations of the Chaco riverside.

Keywords: Late Holocene, archaeological components, riverine subsistence system.

\section{Introducción}

El Gran Chaco sudamericano es un vasto territorio que se extiende desde los

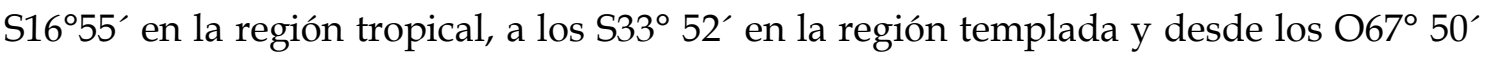
al pie de los Andes, hasta los $057^{\circ} 52^{\prime}$ en la provincia de Corrientes (Naumann 2006). Hasta los comienzos de las investigaciones sistemáticas, la región se ha mantenido como una incógnita en términos arqueológicos. Esto habilitó su caracterización como el agujero negro de la arqueología sudamericana (Braunstein et al. 2002). En la actualidad ha comenzado a revertirse aquella situación permitiendo el planteo de hipótesis regionales y el inicio de estudios intensivos y localizados (Calandra y Salceda 2004; Lamenza et al. 2015). En el caso particular del Chaco argentino se ha propuesto una sectorización preliminar con criterio ecológico cultural que aún resulta operativa a los fines de sistematizar la información (De Feo et al. 2003). Uno de ellos, el Sector Ribereño Paraguay Paraná (SRPP) se incluye dentro de la gran Región Neotropical, Dominio Chaqueño, Provincia Chaqueña (Cabrera y Willink 1973) y se adscribe a los grandes humedales sudamericanos (Neiff et al. 1994).

Sus rasgos característicos son los bosques muy altos a orillas de los ríos; altos con presencia de leñosas de maderas duras y bajos con predominancia de algarrobos. A su vez, se encuentran presentes dos tipos de ambientes graminiformes, el de pastizales no inundables y el de pajonales (Morello y Adámoli 1968). La sucesión de los cursos de agua en vinculación con los sitios arqueológicos detectados (v. gr. Porteño, Monte Lindo, Pilagá, Salado, Guaycurú, de Oro, Negro y Tapenagá) intercalan esteros, cañadas, palmares y selvas en galería.

Los contextos arqueológicos investigados permiten identificar sitios uni y multicomponentes de actividades múltiples, en montículos, que se encuentran distribuidos de a pares sobre elevando el terreno por encima del nivel de inundación e interconectando cursos con cuerpos de agua (Lamenza 2015a). Las trayectorias históricas de estos pueblos incluyen gran diversidad cultural con relaciones de interacción diferenciales dentro y fuera de la región (Lamenza et al. 2015). 


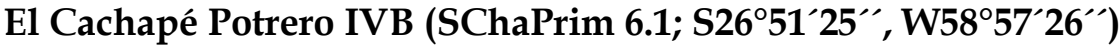

El sitio se encuentra dentro de la Localidad Arqueológica El Cachapé y se presenta como una estructura monticular en relación con un cuerpo de agua secundario (arroyo Tucá), sobre una línea de albardón donde se ha desarrollado un bosque ripario de inundación (sensu Ginzburg y Adámoli 2006; Figura 1A). Esta estructura se encuentra en un sector sobre elevado del terreno y por encima del nivel de inundación en la época de lluvia.
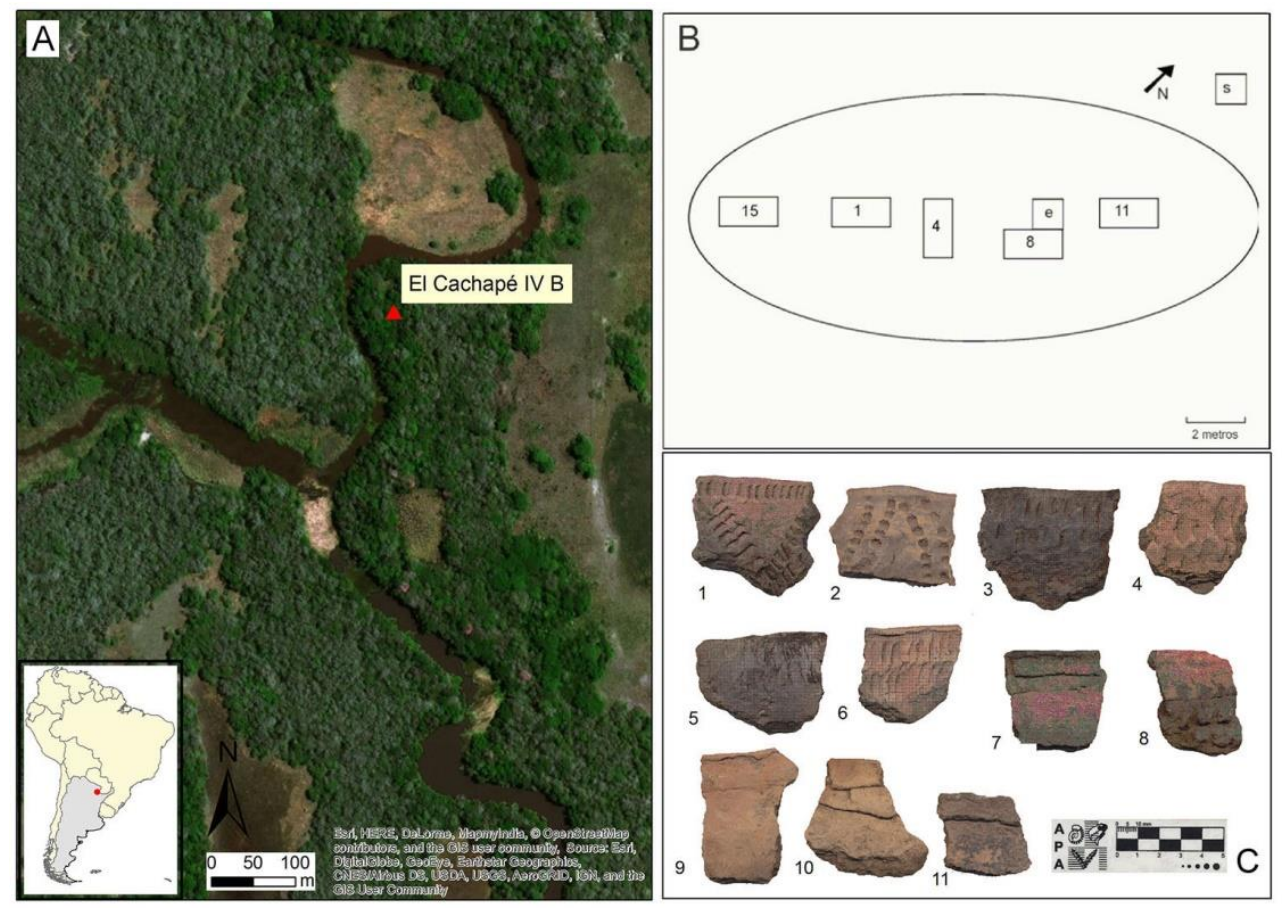

Figura 1. Sitio El Cachapé Potrero IVB. A-Localización, B- Planta del sitio (los números corresponden a las cuadrículas realizadas), C- Fragmentos cerámicos. 1 a 6 representativos del Componente B y 7 a 11 del Componente A.

Los primeros análisis de la arqueofauna (en un trabajo pionero para la zooarqueología de la región) se basaron en una muestra de 745 especímenes (Santini 2009). Teniendo en cuenta la cuantificación del reanálisis, la misma corresponde al $19,74 \%$ del conjunto total actual. En ese momento se analizaron los restos como pertenecientes al lapso de una sola ocupación y se destacó una mayor abundancia relativa de huesos asignados a Peces. Sin embargo, teniendo en cuenta los tamaños de las presas, se consideró que Blastocerus dichotomus fue el principal recurso explotado por los antiguos pobladores, complementado por una mayor explotación del recurso peces y en menor medida de Myocastor coypus (Santini 2009; Figura 2). Se resaltó la menor representación de $M$. coypus como recurso en relación con otros sitios (El Cachapé Potrero V y Sotelo I). El sitio El Cachapé Potrero IVB da cuenta de la existencia de taxa de hábitos acuáticos, y donde los pobladores desarrollaron una economía pescadora, cazadora-recolectora (Santini 2009). 


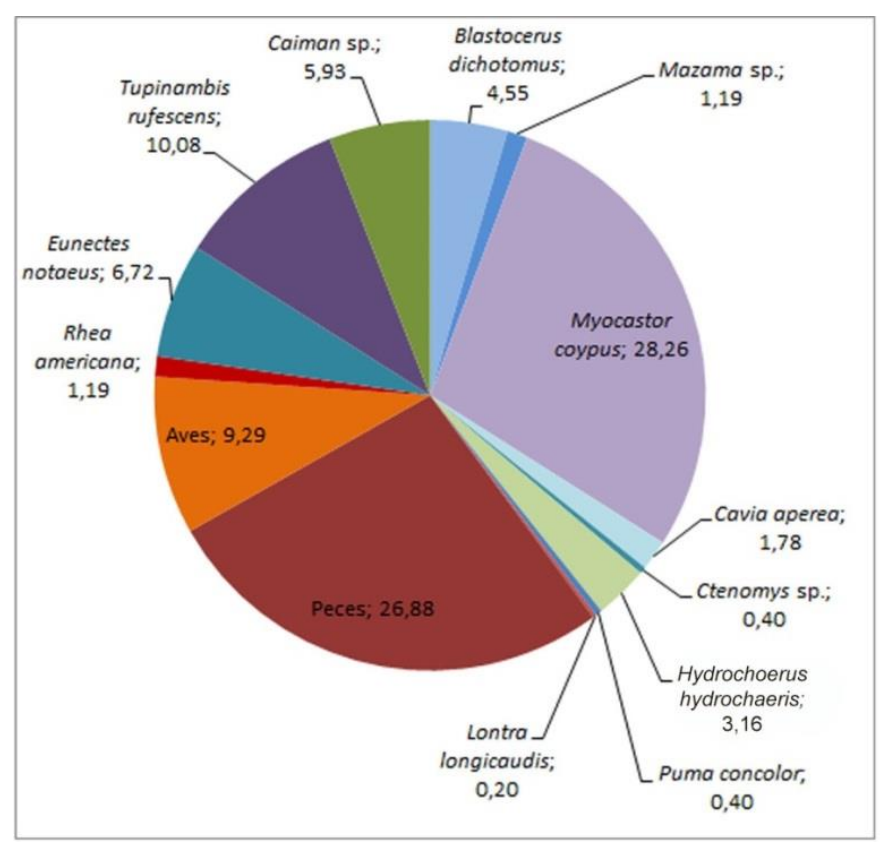

Figura 2. Abundancia taxonómica (NISP\%) del sitio El Cachapé Potrero IVB, basado en los datos obtenidos en el primer análisis (Santini 2009).

El componente cerámico recuperado en el sitio representa las diferentes etapas de la producción (manufactura, uso, reciclado y descarte). En términos generales, las pastas presentan núcleos que van del gris al negro, con márgenes grisáceos y pardos, cocción oxidante incompleta, inclusiones de tiesto molido de tamaño mediano. La técnica de manufactura utilizada ha sido la de rodete. Los fragmentos poseen una dureza media, 3 y 4 de la escala de Möhs (1825), y su fractura es irregular. La matriz presenta una textura media y porosa. Las formas presentes son abiertas no restringidas y restringidas, ambas de contornos simples, como son las escudillas, cuencos y ollas, con diámetros que oscilan entre $12-36 \mathrm{~cm}$. La decoración incluye incisiones de líneas finas y gruesas, de surco rítmico, punteados, presiones unguiculares, impronta de cordelería y, con mayor frecuencia, corrugados, en sus variantes simple, inciso y unguiculado (Lamenza 2013).

Estudios pormenorizados del componente cerámico en el sitio El Cachapé Potrero IVB permitieron complementar la información estratigráfica y cronológica a través de la identificación de distintos agrupamientos (Lamenza 2015b). A partir de los agrupamientos resultantes se profundizó en analizar la diversidad interna del ítem cerámico en el sitio en cuestión. De manera particular, un estudio posterior, permitió diferenciar conjuntos. Uno, denominado Componente A (Figura 3), representado por el material cerámico de las cuadrículas 4 (nivel 0,50-0,60m), 8 (niveles de 0,40 a 0,60m) y 11 (niveles de 0,30 a 0,50m, Figura 1B y C). El otro, Componente B (Figura 3), agrupa los niveles superiores de las cuadrículas 4, 8 y la totalidad de la 15 (Figura 1B y C). Aquí también se integran los materiales recuperados en recolección superficial y los del sondeo estratigráfico. A su vez, dentro de este grupo pueden diferenciarse dos subconjuntos (Figura 3), uno conformado por los niveles intermedios (entre 0,30 y 
0,40m) de las cuadrículas 15 y 8; otro por los fragmentos recuperados en los niveles superiores (entre 0,20 y 0,30m) de la cuadrícula 8 , los hallados entre los 0,40 y $0,50 \mathrm{~m}$ de las cuadrículas 4 y los niveles de 0,40 a $0,60 \mathrm{~m}$ de profundidad de la cuadrícula 15 (Lamenza et al. 2018a).

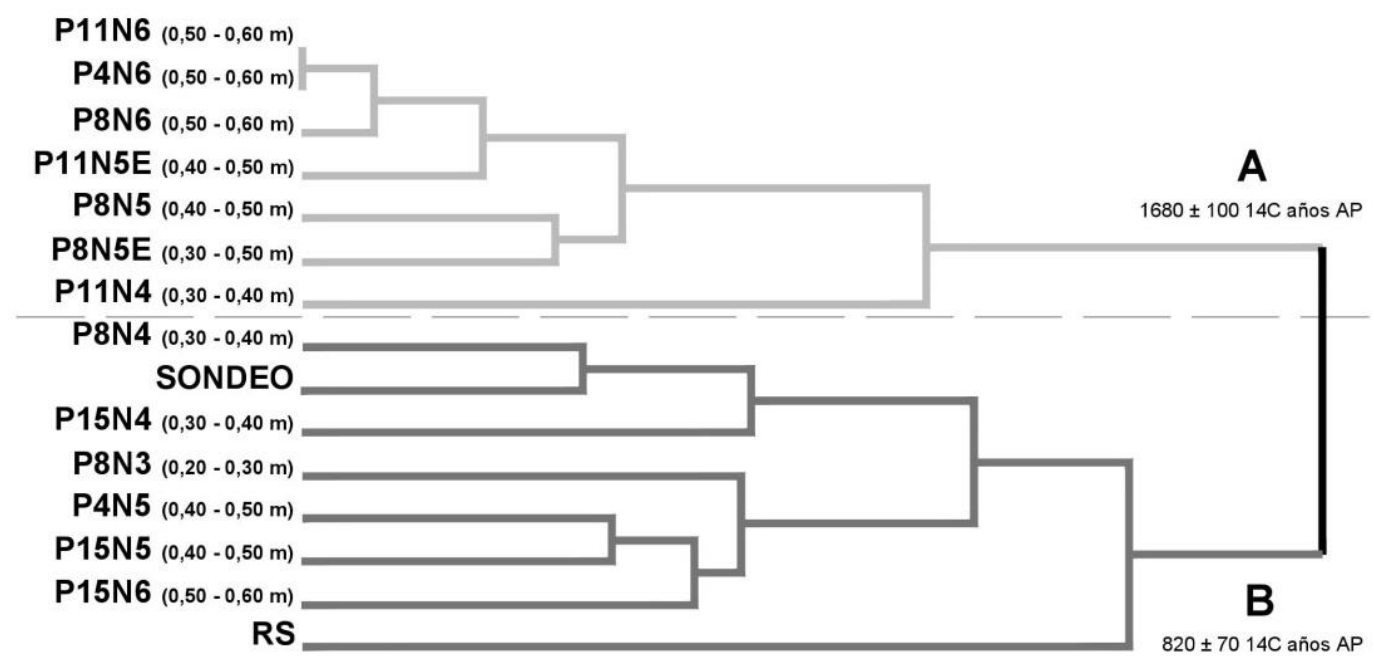

Figura 3. Definición de los Componentes (modificado de Lamenza et al. 2018).

La información provista por la cronología radiocarbónica nos permitió reforzar la validez de los agrupamientos resultantes. A partir de la realización de dos dataciones se puede estimar una antigüedad de $c a .1700$ años AP para el Componente A y de ca. 900 años AP para el Componente B (Lamenza et al. 2018a). Estos avances, desde el análisis cerámico y cronológico, sugieren revisar la información arquefaunística con el objetivo de indagar sobre la variación temporal en la disponibilidad y estructura de los recursos explotados por las antiguas sociedades chaqueñas.

\section{Materiales y métodos}

El conjunto total del sitio presenta 3774 especímenes arqueofaunísticos (óseos, cáscaras de huevo y valvas). Se realizó la determinación anatómica y taxonómica por comparación con colecciones de referencia de la Facultad de Ciencias Naturales y Museo (UNLP) y, cabe aclarar, que debido al grado de fragmentación de los especímenes óseos se ha llegado a distintos niveles jerárquicos. Se diferenció la categoría Mammalia en rangos de tamaño estimados; en este sentido, la categoría mamíferos grandes corresponde a aquellos mayores a los $50 \mathrm{~kg}$ ( $v$. gr. Blastocerus dichotomus -ciervo de los pantanos-), mamíferos medianos entre 15 y $50 \mathrm{~kg}$ ( $v . g r$. Ozotoceros bezoarticus -venado de las pampas-, Hydrochoerus hydrochaeris -carpincho- $\mathrm{y}$ Puma concolor -puma-), mamíferos pequeños entre 1 y $15 \mathrm{~kg}$ (v. gr. Myocastor coypus coipo-) y micromamíferos a aquellos menores a $1 \mathrm{~kg}$ ( $v$. gr. Cavia aperea -cuis-). Cabe aclarar que debido a que la reevaluación de los materiales no fue realizada por los mismos investigadores que hicieron el análisis original, pueden darse diferencias en el 
nivel de determinación debido al adiestramiento del investigador y las diferencias en la formación, así como la posible mayor fragmentación por el manejo de los materiales o técnicas de conservación (factores tréficos).

Para la cuantificación se tuvieron en cuenta los cálculos referentes a la cantidad de especímenes de la muestra (NSP; Grayson 1991), a la abundancia taxonómica en forma de NISP (number of identified specimens per taxón; Payne 1975), \%NISP y MNI (minimum number of individuals; White 1953). En cuanto a la representación de partes anatómicas se realizaron los cálculos de MNE (minimum number of elements; Binford 1984) a través de la identificación de zonas diagnósticas siguiendo a Mengoni Goñalons (1999).

Se analizaron los procesos y agentes tafonómicos que contribuyeron a la formación del registro arqueofaunístico. Se estudiaron las modificaciones de la superficie ósea, las cuales fueron analizadas a nivel macroscópico y con lupa de mano de 15 aumentos. En relación con la preservación de los materiales, se tuvieron en cuenta los estadíos de meteorización siguiendo las propuestas de Andrews (1990), Behrensmeyer (1978) y Behrensmeyer et al. (2003), según el grupo correspondiente (micromamíferos, mamíferos mayores a $5 \mathrm{~kg}$ y aves). Teniendo en cuenta que no se encuentran disponibles escalas de meteorización ósea para peces, anfibios y reptiles, se propone seguir los criterios generales de meteorización propuestos por Behrensmeyer (1978). En este sentido se consideró como estadio 0 a la ausencia de rasgos propios de la meteorización; estadio 1, cuando los especímenes presentaban fisuras; estadio 2 cuando comenzaban a desarrollarse grietas y exfoliación; estadio 3 cuando la meteorización generaba pérdida de material óseo; estadio 4 ,cuando se acrecentaba la superficie del hueso donde se observaban los atributos antes mencionados; y estadio 5, aquellos restos que tienen un estado de preservación pobre y se produce la fragmentación y/o pérdida de material óseo en el manipuleo del material in situ.

También se analizaron los restos con el fin de identificar marcas de depredadores naturales (v. gr. carnívoros, aves rapaces, Andrews 1990, Binford 1981, Blumenschine et al. 1996), las producidas por roedores, las precipitaciones químicas y las ocasionadas por la acción de raíces (Lyman 1994). Por otra parte, se analizaron las modificaciones de origen antrópico, que incluyen: marcas de corte, machacado, raspado, percusión, fractura intencional, termoalteración y evidencias de formatización (v. gr. Binford 1981, Blumenschine et al. 1996, Mengoni Goñalons 1999, Scheinsohn 1997, Shipman et al. 1984).

Con el fin de comparar las ocupaciones identificadas a través del análisis cerámico y los fechados radiocarbónicos, se discriminaron los materiales arqueofaunísticos siguiendo la procedencia estratigráfica identificada para cada componente. De esta manera, no se incluyeron en el análisis aquellos materiales que no pudieron asignarse a alguna ocupación en particular, dando como resultado una reducción del conjunto (NSP=2222). Para el Componente A se analizaron un total de 1446 especímenes y para el Componente B de 776. La comparación se realizó mediante los índices de Riqueza, Diversidad, Equitatividad y abundancia relativa a través del NISP como unidad. 
Índice de Riqueza: refleja la cantidad de taxones utilizados en un sitio (Grayson y Delpech 1993), siendo la sumatoria de todas las categorías taxonómicas que no se traslapen (Grayson 1991). Expresado como NTAXA.

Índice de Diversidad: mide la importancia relativa de las especies presentes, permite la discusión de las estrategias de subsistencia en términos de la variedad de animales usados en un sitio. Este será calculado con el índice de Shanon-Weaver (Reitz y Wing 1999). El mismo se expresa como $H^{\prime}=-\sum$ (pi) (ln pi); donde pi es el número de especímenes de la categoría $i$ dividido el tamaño de la muestra y $\ln$ es logaritmo natural.

Índice de Equitatividad: mide la igualdad con que las especies fueron utilizadas, el grado de dependencia de los recursos utilizados y la variedad de especies usadas en el sitio basado en el uso igual o desigual de las mismas (Reitz y Wing 1999). Se utilizará la formula $V^{\prime}=H^{\prime}$ / In $S$; donde $H^{\prime}$ es el índice de Shanon-Weaver, y $S$ es la cantidad de taxones (NTAXA).

Estos índices son dependientes del tamaño de la muestra (Grayson et al. 2001) por lo que para poder comparar muestras de diferentes tamaños, los cálculos de Riqueza y Diversidad fueron realizados por el software EcoSim 700 mediante curva de rarefacción (Lyman y Ames 2007). Además, al utilizar el NISP como unidad para calcular estos índices, también entra en juego la fragmentación de las muestras que puede incidir en este cálculo (v. gr. Grayson 1984; Lyman 1994; Reitz y Wing 1999). Por ello se calculó el índice de fragmentación NSP/NISP (Grayson 1991), sin tener en cuenta los elementos enteros (Lyman 1994). Para realizar los índices de Diversidad y Equitatividad se agruparon los NISP de las distintas categorías de peces y no se tuvieron en cuenta las categorías abarcativas (v. gr. Squamata, Mammalia indet. grande, mediano, pequeños y micro- y Cervidae).

Índice de abundancia relativa: en esta oportunidad se tendrá en cuenta el índice de fauna mayor considerando los especímenes de mamíferos medianos y grandes en relación al conjunto de cada muestra y se puede expresar como NISP mamíferos medianos + NISP de mamíferos grandes/NISP de la muestra.

\section{Resultados}

En ambos componentes (Tabla 1) se observa un predominio de los peces a través del NISP, seguido por M. coypus. Cabe destacar que a través del MNI se observan valores similares entre los taxones representados de ambos componentes (con predominancia de peces, seguido por $M$. coypus y luego las aves, Tabla 1). Teniendo en cuenta que el cálculo de MNI en muestras pequeñas hace más proporcionada la relación entre algunos de los recursos, se decidió utilizar el NISP y su porcentual para estimar las diferencias entre los conjuntos. 
Componente A

\section{Abundancia taxonómica}

En la Tabla 1 y Figura 4A se observa que en el Componente A existe una preponderancia de los peces (incluidos los restos asignados a Lepidosiren paradoxa, Characiformes, Siluriformes, Loricariidae y Symbranchus marmoratus), seguido por $M$. coypus, luego en proporciones similares las aves, Squamata (incluyendo Boidae y Salvator sp.), Cervidae y Gastropoda, en menor proporción H. hydrochaeris, Caiman sp. y C. aperea, siendo escasos los restantes recursos. Es preciso mencionar que teniendo en cuenta el tamaño de los cérvidos, corresponderían a los recursos de mayor importancia en cuanto a la cantidad de nutrientes.

\begin{tabular}{|c|c|c|c|c|c|c|c|c|c|c|}
\hline \multirow[b]{2}{*}{ Taxón } & \multicolumn{5}{|c|}{ Componente A } & \multicolumn{5}{|c|}{ Componente B } \\
\hline & NISP & $\begin{array}{c}\text { NISP } \\
\%\end{array}$ & MNE & $\begin{array}{c}\mathrm{N} \\
\text { Ent. }\end{array}$ & MNI & NISP & $\underset{\%}{\mathrm{NISP}}$ & MNE & $\begin{array}{c}\mathrm{N} \\
\text { Ent. }\end{array}$ & MNI \\
\hline Gastropoda & 66 & 7,53 & - & - & - & 14 & 2,8 & - & - & - \\
\hline Teleostei ${ }^{*}$ & 222 & 25,31 & 135 & 9 & 15 & 246 & 49,2 & 152 & 23 & 32 \\
\hline Anura & - & - & - & - & - & 1 & 0,2 & 1 & 1 & - \\
\hline Rhinella sp. & - & - & - & - & - & 1 & 0,2 & 1 & - & 1 \\
\hline Caiman sp. & 12 & 1,37 & 10 & 2 & 1 & 10 & 2 & 6 & 1 & 1 \\
\hline Squamata & 6 & 0,68 & 5 & - & - & 12 & 2,4 & 5 & - & - \\
\hline Salvator sp. & 5 & 0,57 & 5 & - & 1 & 4 & 0,8 & 4 & 2 & 1 \\
\hline Boidae & 47 & 5,36 & 38 & 28 & 1 & 14 & 2,8 & 14 & 14 & 1 \\
\hline Aves & 54 & 6,16 & 36 & 6 & 3 & 24 & 4,8 & 14 & 3 & 2 \\
\hline Rheidae (cáscaras) & 1 & 0,11 & - & - & - & - & - & - & - & - \\
\hline Mammalia indet. & 4 & 0,46 & - & - & - & - & - & - & - & - \\
\hline $\begin{array}{l}\text { Mammalia } \\
\text { (grande) }\end{array}$ & 110 & 12,54 & 12 & - & - & 17 & 3,4 & 2 & - & - \\
\hline Cervidae & 9 & 1,03 & 2 & - & - & 2 & 0,4 & 2 & - & - \\
\hline $\begin{array}{l}\text { Blastocerus } \\
\text { dichotomus }\end{array}$ & 32 & 3,65 & 15 & - & 1 & 2 & 0,4 & 2 & - & 1 \\
\hline $\begin{array}{l}\text { Mammalia } \\
\text { (mediano) }\end{array}$ & 107 & 12,20 & 9 & 1 & - & 27 & 5,4 & 5 & - & - \\
\hline $\begin{array}{l}\text { Ozotoceros } \\
\text { bezoarticus }\end{array}$ & 13 & 1,48 & 9 & 1 & 2 & 2 & 0,4 & 2 & - & 1 \\
\hline Puma concolor & 1 & 0,11 & 1 & 1 & 1 & - & - & - & - & - \\
\hline $\begin{array}{l}\text { Hydrochoerus } \\
\text { hydrochaeris }\end{array}$ & 25 & 2,85 & 6 & 2 & 1 & 11 & 2,2 & 2 & - & 1 \\
\hline $\begin{array}{l}\text { Mammalia } \\
\text { (pequeño) }\end{array}$ & 18 & 2,05 & 9 & 2 & - & 26 & 5,2 & 3 & - & - \\
\hline Marsupialia & - & - & - & - & - & 3 & 0,6 & 1 & - & 1 \\
\hline Carnivora & 1 & 0,11 & - & - & - & - & - & - & - & - \\
\hline Myocastor coypus & 130 & 14,82 & 38 & 4 & 5 & 76 & 15,2 & 18 & 8 & 3 \\
\hline Mammalia (micro) & 3 & 0,34 & 2 & - & - & 1 & 0,2 & 1 & - & - \\
\hline Cavia aperea & 11 & 1,25 & 7 & 1 & 2 & 7 & 1,4 & 6 & 1 & 1 \\
\hline Total & 877 & 100,00 & & 56 & & 500 & 100,00 & & 39 & \\
\hline Indet. & 569 & & \multicolumn{3}{|c|}{$\begin{array}{l}\text { NSP/ } \\
\text { NISP }\end{array}$} & 276 & & \multicolumn{3}{|c|}{ NSP/ } \\
\hline NSP & 1446 & & & 1,69 & & 776 & & & 1,59 & \\
\hline
\end{tabular}

Tabla 1- Abundancia taxonómica e índices de fragmentación. 
$\mathrm{N}$ Ent. = número de restos enteros; * Desglose de los peces, componente $\mathrm{A}=$ Lepidosiren paradoxa, Characiformes, Siluriformes, Loricariidae y Symbranchus marmoratus; Componente $\mathrm{B}=$ Lepidosiren paradoxa, Characiformes, Serrasalmus sp., Siluriformes, Loricariidae y Symbranchus marmoratus.

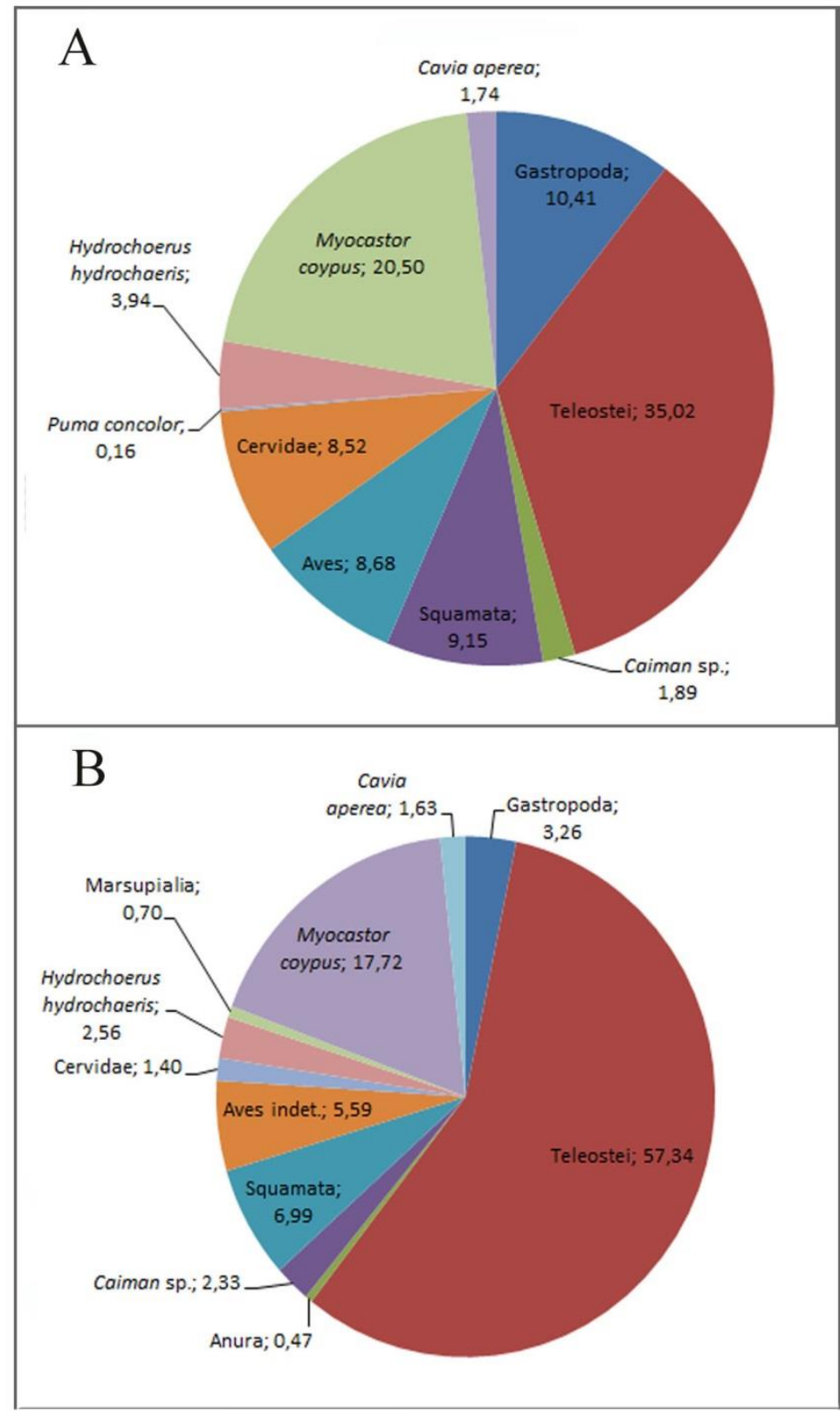

Figura 4. Abundancia taxonómica (NISP\%). A- Componente A; B- Componente B.

\section{Modificaciones óseas}

Con respecto a la preservación de la muestra del Componente A, en las distintas categorías analíticas se observa un predominio de especímenes no meteorizados y en estadios bajos (E1 y en menor medida E2), mientras que escasos restos presentan meteorización más avanzada (E3, Figura 5A). Sólo en la categoría de mamíferos mayores a $5 \mathrm{~kg}$ se observa un patrón un poco diferente, predominando el estadio 1, 
seguido por el 0 , con una mayor proporción de los estadios más avanzados respecto a los restantes grupos, pero en general, consideramos que la muestra se enterró relativamente rápido. Entre las modificaciones naturales se destacan las precipitaciones de óxido de manganeso en el 40,24\% de los restos, las cuales son características de ambientes húmedos y con fluctuación de la capa freática (Tabla 2). Por otra parte, las marcas de raíces y roedores son escasas y no se registró la presencia de atributos asignables a depredadores naturales (v. gr. marcas de mordisqueo y/o corrosión gástrica, Tabla 2).

A

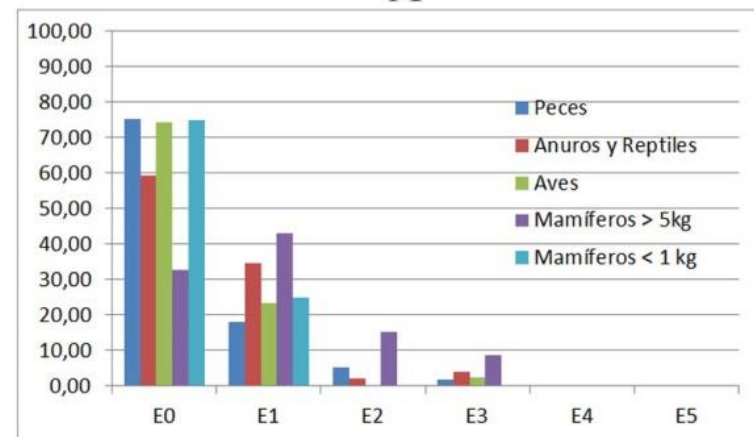

B

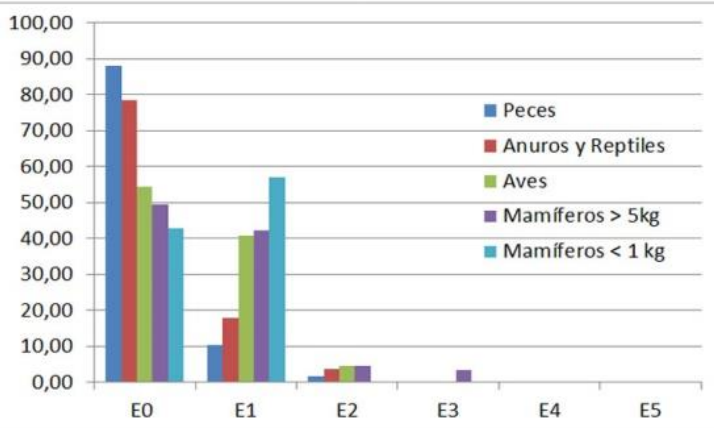

Figura 5- Perfil de meteorización. A- Componente A; B- Componente B.

\begin{tabular}{|l|c|c|}
\hline & Componente A & Componente B \\
\hline Raíces & $2,35 \%$ & $4,51 \%$ \\
$\begin{array}{l}\text { Precipitaciones } \\
\text { (manganeso) }\end{array}$ & $40,24 \%$ & $56,31 \%$ \\
Roedores & $0,13 \%$ & $0,38 \%$ \\
Carnívoros- aves rapaces & - & - \\
\hline
\end{tabular}

Tabla 2- Modificaciones de origen natural por componente.

Con respecto a la acción antrópica, se observó una escasa proporción de marcas de corte (Tabla 3). Tanto en peces, aves y M. coypus se identificaron posibles marcas de corte (para confirmarlas será necesario analizarlas en microscopio electrónico de barrido con el fin de observar las estrías internas). En peces se registró en el cuerpo de una vértebra, en las aves sobre una diáfisis indeterminada (tres incisiones superficiales) y en $M$. coypus en la cara ventral del cuerpo de una vértebra lumbar. La restante marca de corte se observó en un fragmento indeterminado. Por otra parte, la mayor proporción de restos termoalterados se presentan en los cérvidos (Cervidae, $B$. dichotomus y $O$. bezoarticus), seguido por las categorías abarcativas de Mammalia indet. grande (posibles restos de B. dichotomus), pequeños y medianos (Tabla 3). En escasa proporción se observan restos termoalterados en peces (Teleostei y Siluriformes), $M$. coypus y restos indeterminados (Tabla 3). La fractura fresca sólo se identificó en escasa proporción en huesos largos de Mammalia indet. grande y se registró el uso de materia 
prima para la confección de artefactos en Mammalia indet. mediano e indeterminados (Tabla 3). La tecnofractura se presentó sobre un fragmento de diáfisis de mamífero mediano con un borde pulido, y dos puntas (una plana y un fragmento de punta cóncavo-convexa), que por su modificación en la formatización, dificultó asignarlos a un grupo taxonómico.

\begin{tabular}{|c|c|c|c|c|c|c|c|c|c|c|c|}
\hline \multicolumn{12}{|l|}{ Componente A } \\
\hline Taxón & $\mathrm{N}$ & Co & $\%$ & Q & $\%$ & HL & $\underset{\mathrm{F}}{\mathrm{FK}}$ & $\%$ & NL & Art & $\%$ \\
\hline Teleostei & 114 & $1 ?$ & 0,87 & 1 & 0,88 & - & - & - & - & - & - \\
\hline Siluriformes & 85 & - & - & 2 & 2,35 & - & - & - & - & - & - \\
\hline Aves & 54 & $1 ?$ & 1,85 & - & - & - & - & - & - & - & - \\
\hline $\begin{array}{l}\text { Mammalia indet. } \\
\text { (grande) }\end{array}$ & 110 & - & - & 7 & 6,36 & 68 & 2 & 2,94 & - & - & - \\
\hline Cervidae & 9 & - & - & 1 & 11,11 & - & - & - & - & - & - \\
\hline $\begin{array}{l}\text { Blastocerus } \\
\text { dichotomus }\end{array}$ & 32 & - & - & 6 & 18,75 & - & - & - & - & - & - \\
\hline $\begin{array}{l}\text { Mammalia indet. } \\
\text { (mediano) }\end{array}$ & 107 & - & - & 4 & 3,74 & - & - & - & - & 1 & 0,93 \\
\hline $\begin{array}{l}\text { Ozotoce } \\
\text { bezoarti }\end{array}$ & 13 & - & - & 1 & 7,69 & - & - & - & - & - & - \\
\hline $\begin{array}{l}\text { Mammalia indet. } \\
\text { (pequeño) }\end{array}$ & 18 & - & - & 1 & 5,56 & - & - & - & - & - & - \\
\hline Myocastor coypus & 130 & $1 ?$ & 0,76 & 1 & 0,77 & - & - & - & - & - & - \\
\hline Indet. & 569 & 1 & 0,17 & 1 & 0,18 & - & - & - & - & 2 & 0,35 \\
\hline \multicolumn{12}{|l|}{ Componente B } \\
\hline Taxón & $\mathrm{N}$ & Co & $\%$ & $\bar{Q}$ & $\%$ & $\overline{\mathrm{HL}}$ & $\begin{array}{c}\mathrm{FK} \\
\mathrm{F}\end{array}$ & $\%$ & $\mathrm{NL}$ & $\overline{\text { Art }}$ & $\%$ \\
\hline Teleos & 90 & - & - & 1 & 1,11 & - & - & - & - & - & - \\
\hline Caiman sp. & 10 & - & - & 2 & 20 & - & - & - & - & - & - \\
\hline Squamata & 12 & - & - & 2 & 16,67 & - & - & - & - & - & - \\
\hline Aves & 24 & 2 & 8,33 & - & - & - & - & - & - & - & - \\
\hline $\begin{array}{l}\text { Mammalia indet. } \\
\text { (grande) }\end{array}$ & 17 & - & - & 2 & 11,76 & 16 & 5 & 31,25 & 1 & - & - \\
\hline Cervidae & 2 & - & - & - & - & - & - & - & - & 2 & 100 \\
\hline $\begin{array}{l}\text { Blastocerus } \\
\text { dichotomus }\end{array}$ & 2 & - & - & 1 & 50 & - & - & - & - & - & - \\
\hline $\begin{array}{l}\text { Mammalia indet. } \\
\text { (mediano) }\end{array}$ & 27 & - & - & 5 & 18,52 & 21 & 5 & 23,8 & - & 2 & 7,4 \\
\hline $\begin{array}{l}\text { Mammalia indet. } \\
\text { (pequeño) }\end{array}$ & 26 & - & - & 3 & 11,54 & - & - & - & - & - & - \\
\hline Myocastor coypus & 76 & $1 ?$ & 1,31 & - & - & - & - & - & - & - & - \\
\hline Indet. & 276 & 1 & 0,36 & 14 & 5,07 & - & - & - & - & 1 & 0,36 \\
\hline
\end{tabular}

Tabla 3- Modificaciones antrópicas por componente. $\mathrm{Co}=$ marcas de corte; $\mathrm{Q}=$ termoalteración; $\mathrm{HL}=$ cantidad de huesos largos; $\mathrm{FK} \mathrm{F}=$ fracturas frescas; $\mathrm{NL}=$ negativos de lascado; Art= tecnología ósea.

Diversidad, Equitatividad y Abundancia Relativa

Teniendo en cuenta los aspectos tafonómicos y los antecedentes generados para la región (Santini 2009), para los cálculos de diversidad, equitatividad y abundancia relativa, se tuvieron en cuenta los taxones con evidencias de consumo antrópico (siendo estos escasos) y aquellos que por su frecuencia y la ausencia de indicadores de incorporación natural, no se descartan del consumo humano. En este sentido, para el 
Componente A se consideraron todas las categorías taxonómicas. Se aclara, que en el caso de C. aperea si bien su frecuencia es baja, los especímenes presentan las mismas características tafonómicas que los restantes taxones, no se registró la acción de depredadores ni restos articulados, y esta especie ha presentado evidencias o se ha postulado el consumo antrópico para sitios de la región (El Cachapé Potrero V, Sotelo I y Puesto Fantín, Santini 2009). Teniendo en cuenta estas consideraciones no los descartamos del consumo humano. Por otra parte, si bien el especímen de $P$. concolor corresponde a un fragmento de canino, y su presencia podría deberse a aspectos simbólicos o al uso de sus pieles (Santini 2009: 174), teniendo en cuenta la gran cantidad de restos fragmentarios asignados a mamíferos medianos, no descartamos que la representación de partes referentes al consumo de nutrientes esté subestimada. La frecuencia en que están representados los distintos taxones en el Componente A es relativamente proporcionada (Figura 4A), obteniéndose un NTAXA de 16, un índice de Diversidad de 1,91 y de Equitatividad de 0,74. El índice de Fauna Mayor presenta un valor de 0,34 .

\section{Componente B}

Abundancia taxonómica

Por otra parte, en el Componente B se observa una clara predominancia de los peces (incluidos los restos asignados a L. paradoxa, Characiformes, Serrasalmus sp., Siluriformes, Loricariidae y S. marmoratus), seguido por M. coypus, con una baja proporción de aves, Gastropoda, C. aperea, H. hydrochaeris, Cervidae, Caiman sp. y Boidae, siendo los restantes recursos escasos (Figura 4B).

Modificaciones óseas

La preservación de la muestra del Componente $B$ en general es buena, destacándose en el perfil de meteorización, la preponderancia del estadio 0, seguido por el estadio 1 y siendo escasos los restos con meteorización avanzada en las distintas categorías analíticas (Figura 5B). Si bien se observan diferencias entre los taxones (Figura 5B), tal vez, propio de las características intrínsecas a nivel de Clase y tamaño, se interpreta un enterramiento relativamente rápido de los restos. Con respecto a las restantes modificaciones naturales, esta muestra sigue las tendencias del Componente A, destacándose las precipitaciones de óxido de manganeso con el 56,31\% de los restos, la escasez de marcas de raíces y roedores ( $4,51 \%$ y $0,38 \%$ respectivamente) y la ausencia de atributos asignables a depredadores naturales (v.gr. marcas de mordisqueo y/o corrosión gástrica, Tabla 2).

Con respecto a las modificaciones antrópicas, en el Componente B se observa una escasa proporción de marcas de corte. Entre ellas, una porción distal de la diáfisis de ulna y de un tibio-tarso de ave presentan incisiones, posiblemente producto de la desarticulación. Al igual que en el Componente $\mathrm{A}$, una vértebra lumbar de $M$. coypus 
presenta una probable marca de corte sobre la cara ventral del cuerpo, evidenciando un posible patrón de evisceración. Por último, un fragmento indeterminado presenta varias incisiones, largas y superficiales. Por otra parte, si bien la proporción de restos termoalterados en el Componente B es mayor que en el A, esto podría estar en relación a la sobredimensión de este cálculo por el menor tamaño de la muestra (Tabla 3). Entre los taxones termoalterados se destacan B. dichotomus, los reptiles (Caiman sp. y Squamata), categorías Mammalia indet. grandes, medianos y pequeños, los restos indeterminados y en bajo número los peces (Tabla 3). En mamíferos medianos y grandes se registraron fracturas frescas y sólo en mamíferos grandes se evidenció la fractura intencional por el registro de un negativo de lascado (Tabla 3). Además, cabe mencionar la presencia de artefactos óseos, un bisel y una punta cóncava-convexa con perforación en su zona mesial sobre asta de Cervidae, dos fragmentos diafisiarios de mamíferos medianos con uno de sus bordes pulidos y un extremo distal de lezna sobre un espécimen indeterminado (Tabla 3).

Diversidad, Equitatividad y Abundancia Relativa

Para el Componente B, en el caso de los anuros y marsupiales, si bien no se registraron evidencias naturales de acumulación que nos permita descartarlos del consumo humano, debido a su escasa frecuencia y a la ausencia de antecedentes sobre evidencias de acumulación antrópica para estos taxones en la región, se decidió descartarlos de los cálculos de diversidad, equitatividad y abundancia relativa. En este caso, la frecuencia en que están representados los distintos taxones es más desproporcionada que en el Componente A, con un NTAXA, índices de Diversidad, Equitatividad y de Fauna Mayor con valores menores $\left(\mathrm{NTAXA}=13, \mathrm{H}^{\prime}=1,36\right.$ y V'= 0,56; Fauna mayor=0,12).

Comparación

A pesar que los tamaños de las muestras son disímiles, (NISP Componente $A=877$; NISP del Componente $B=500$ ), se tomaron los recaudos necesarios con el fin de compararlos. En este sentido, el índice de fragmentación NSP/NISP, si bien más alto en el Componente A, presentan valores similares (Tabla 1). Como se mencionó en el apartado de metodología, los cálculos de Riqueza y Diversidad fueron realizados mediante curva de rarefacción con el fin de tener un control sobre el tamaño de la muestra. En este sentido se puede mencionar que el Componente A es más diverso y equitativo, resultando en una estrategia más generalista de obtención de recursos, donde si bien los recursos de menor tamaño son los preponderantes, la fauna mayor tuvo un rol más destacado que en el Componente B, sobre todo teniendo en cuenta el mayor aporte de recursos por individuo. 


\section{Discusiones}

Para ordenar la discusión vamos a considerar distintos aspectos. En primer lugar establecer las diferencias entre el análisis preliminar (Santini 2009) y los resultados presentados en esta oportunidad. En segundo lugar, profundizar en esta nueva información y dar cuenta de las variaciones encontradas entre ambos componentes. Para finalizar proponer una interpretación preliminar de la variación temporal considerando la cronología y los cambios ambientales en el marco de un proceso histórico integral chaqueño.

Las diferencias del análisis preliminar y los componentes identificados en esta oportunidad se deben a varios factores. En primer lugar debe tenerse en cuenta el aumento de la muestra y la división de los restos faunísticos en dos ocupaciones. A su vez considerar diferencias inter-observador y la posible fragmentación producto del manejo de los materiales en el análisis y conservación por parte de los investigadores. Con respecto a las diferencias inter-observador, puede mencionarse que en el primer análisis se produce una determinación más precisa de los peces, obteniendo 8 taxones (a nivel de género y especie) en contraposición a los 4 determinados en este trabajo. Otra diferencia corresponde a la determinación de los restos de Boidae a nivel de especie, Eunectes notaeus, en el primer análisis y a nivel de familia en esta oportunidad. Asimismo, puede observarse la baja representación de Salvator sp. (sinonimia de Tupinambis) en el reanálisis, esto puede ser debido a la fragmentación en el manejo y conservación de los materiales, ya que registramos una gran cantidad de vértebras fragmentadas que fueron asignadas al orden Squamata. A su vez se decidió asignarlos a nivel de género (Salvator), ya que por restos fragmentarios del esqueleto es muy difícil diferenciar las especies de lagartos, sumado a que en la región se encuentran dos especies en simpatría (Brizuela 2010; Porini 2006). También preferimos mantener una postura conservadora con respecto a los restos asignados en la primera oportunidad como Mazama sp. por tratarse principalmente de fragmentos de asta modificados como artefactos, por lo que se registraron como Cervidae. Por otra parte, el resto asignado a Lontra longicaudis en el primer estudio, consideramos que podría corresponderse con una mandíbula fragmentada y edéntula asignada en esta oportunidad a Carnivora. Por último, los restos de Ctenomys sp. del análisis preliminar, actualmente podrían estar considerados dentro de la categoría Mammalia indet. (micro) debido a procesos de fragmentación por factores tréficos (véase Mengoni Goñalons 1988).

En cuanto a las variables tafonómicas, en ambos componentes se identifican las mismas tendencias. Una buena preservación de los conjuntos, predominio de ausencia de meteorización o estadios bajos, escasa proporción de raíces y roedores, y ausencia de acción de depredadores naturales. Se destacan las precipitaciones de óxido de manganeso, típico del ambiente de depositación donde se encontraron los materiales, asociados a condiciones húmedas o alternancia de la capa freática. En relación con las 
evidencias de procesamiento humano, ambos componentes presentan baja proporción de marcas asignadas a la acción antrópica. Sin embargo, se destacan para el Componente A posibles marcas de corte en aves, peces y $M$. coypus, la termoalteración en cérvidos, categorías abarcativas de mamíferos (grandes, medianos y pequeños) y en escasa proporción en peces y M. coypus. También se registró la confección de artefactos óseos en mamíferos medianos y restos indeterminados. Con respecto al Componente B, se observaron marcas de corte en aves y posibles marcas de corte en M. coypus. La termoalteración en $B$. dichotomus, reptiles (Caiman sp. y Squamata), categorías abarcativas de mamíferos y en escasa proporción los peces. Se evidenció la fractura intencional de los huesos en la categoría mamíferos grandes. Entre los artefactos óseos pudieron asignarse restos a cérvidos, mamíferos medianos y una lezna no pudo determinarse taxonómicamente. En este sentido considerando ambos componentes, se observa una tendencia en el uso de peces, aves, cérvidos y M. coypus. Cabe destacar que la totalidad de los restos faunísticos se encuentran en asociación directa con fragmentos de cerámica que, a su vez, no presentan evidencias de depositación secundaria. Por lo tanto a pesar de la baja frecuencia de evidencias vinculadas al procesamiento humano, consideramos que la mayoría de los taxones representados se corresponden a un aprovechamiento antrópico. Para el Componente B, sólo los anuros y marsupiales, han sido descartados de los cálculos posteriores, considerando que su ingreso posiblemente se deba a cuestiones naturales, teniendo en cuenta su escasa frecuencia y a la ausencia de antecedentes sobre evidencias de acumulación antrópica para estos taxones en la región.

En otro orden de análisis, cabe mencionar algunas consideraciones en relación con los componentes identificados. En términos generales, del análisis tafonómico y en función de los recursos utilizados podemos afirmar que ambos componentes no se diferencian de manera significativa entre sí. El análisis integral del sitio permite establecer que ambos componentes representan desarrollos de actividades múltiples. Todos los recursos son propios de ambientes de humedales, ya sean loticos o lenticos, con mayor o menor caudal y escorrentía diferencial, respectivamente. Por lo tanto el aspecto a destacar en esta oportunidad no es la presencia/ausencia de taxones en los componentes sino la tendencia a lo largo del tiempo en la proporción de los recursos explotados. De manera específica, a la disminución en el consumo de cérvidos y al aumento en la explotación de peces. En términos de estrategias de obtención de recursos consideramos que los peces son de acceso directo en las inmediaciones del sitio de ocupación, mientras que para la captura de cérvidos es necesario implementar partidas de caza a mayores distancias. Por lo tanto proponemos que la principal diferencia entre ambos componentes se debe a una reducción del área de captación de los recursos a lo largo del tiempo.

Esta tendencia puesta en relación con la información cronológica y ambiental permite profundizar en el modelo de subsistencia regional. El marco ambiental de estas ocupaciones puede caracterizarse siguiendo el modelo de Iriondo (2006) el cual consideramos que, hasta el momento, es el más aproximado para comprender las 
variaciones climáticas chaqueñas. El Componente A cuenta con una datación de $1680 \pm 100{ }^{14} \mathrm{C}$ años AP (LP1734, óseo faunístico) lo que permite vincularlo con el Periodo Seco del Holoceno (PSH, 3500-1400 años AP) caracterizado por un clima seco y semiárido (Iriondo 2006).

Esto a priori podría plantear una situación problemática al momento de interpretar el registro del Componente A dado que, como hemos mencionado anteriormente, la fauna recuperada está en estrecha vinculación con recursos ribereños. Para ello, proponemos algunas alternativas. Por un lado, podemos considerar que el PSH no se manifestó con la misma intensidad en este sector específico como se propone para el resto de la llanura chaco-pampeana. Esta situación ya ha sido planteada a raíz de las dataciones radiocarbónicas más tempranas vinculadas a la entidad Goya Malabrigo en el ámbito de la llanura aluvial del Paraná (Echegoy 1994). Sin lugar a dudas los recursos explotados no dan cuenta de un ambiente seco y semiárido al momento de la primera ocupación del sitio aunque esta situación no es condición sine qua non para poner en duda la extensión del PSH en el Sector Ribereño Paraguay Paraná.

En este sentido también recordamos que Iriondo (2006) plantea que las variaciones ambientales en el Chaco deben ser entendidas como fluctuaciones de este a oeste de los límites Chaco seco/húmedo. O sea que para interpretar mejor el PSH debemos considerarlo como un corrimiento hacia el este del Chaco occidental y también tener en cuenta que los grandes ríos “...constituyeron importantes corredores y refugios del ecosistema y de la población humana vinculada al mismo..." (Iriondo 2006: 46). Por lo tanto, dado que el sitio se encuentra en el extremo oriental chaqueño y en vinculación directa con un tributario próximo a su desembocadura en el Río Paraguay, tiene sentido la presencia de recursos ribereños aunque ésta se deba a una situación de refugio dentro de un ámbito generalizado de sequía. Al mismo tiempo considerar los cursos principales como corredores es coherente con el marco cronológico y la dinámica poblacional propuesta en la actualidad. Las primeras evidencias de ocupación humana en el Chaco Húmedo se encuentran dentro de este periodo y se han podido establecer esferas de interacción que incluyen el Chaco Boreal, Pantanal y Mato Grosso do Sul (Lamenza et al. 2018b).

Estas primeras ocupaciones se producen por desplazamientos de norte a sur de sociedades de fuerte vinculación ribereña. A esto último puede sumarse la consideración de eventos extraordinarios (ENOS), como el identificado hace 1500 años AP (Meggers 1994), los cuales tuvieron alcance continental diferencial en distintas regiones. Tal vez estos movimientos poblacionales se hayan visto favorecidos por el incremento de los refugios ribereños debido al aumento de las precipitaciones en la región chaqueña. En contraste, la periferia se ve afectada por condiciones más extremas de sequía y semiaridez. Por lo tanto para este momento, estimamos que la disponibilidad de recursos ribereños se encuentra restringida y localizada. La explotación de recursos está orientada a los cursos de agua en un contexto de baja densidad poblacional con mayor rango de acción. 
Finalmente, el componente B con un fechado de $820 \pm 70{ }^{14} \mathrm{C}$ años AP (LP2506, óseo faunístico), se encuentra en la última etapa del Máximo Térmico Medieval, periodo caracterizado por un aumento generalizado de la humedad con clima tropical y abundantes lluvias sobre todo el Chaco (Iriondo 2006). A su vez, se complejiza la red hidrográfica, avanza la selva, los bosques y los parques arbóreos (Popolizio 2006). Esta situación permite predecir un aumento en la disponibilidad de recursos y estimar transformaciones en la estructura poblacional del área, modificando la territorialidad y la organización de las bandas. Para este momento el Chaco prehispánico se encuentra ocupado de manera generalizada y, como hemos visto, en este componente aumenta la tendencia hacia la pesca y reducción en la explotación de cérvidos. Por lo tanto, proponemos una estrategia de menor rango de acción en un marco de mayor disponibilidad ambiental condicionada por un aumento generalizado de la población a nivel regional.

\section{Consideraciones finales}

El avance en las investigaciones de los materiales arqueológicos del sito El Cachapé Potrero IVB posibilitó la diferenciación de al menos dos momentos de ocupación (Componente A y B). Esto habilitó el reanálisis de la arqueofauna, estimando una diferenciación en la proporción de los recursos a través del tiempo. Estudios próximos nos permitirán profundizar sobre el uso y apropiación de los recursos (v. gr. tamaño de los peces y análisis de representación de partes anatómicas). A su vez, estudios complementarios sobre tecnología ósea, restos vegetales, isótopos estables, entre otros, contribuirán al conocimiento del pasado de las ocupaciones del sitio. Incluso, se plantea la posibilidad de encarar otra estrategia de excavación con el fin de identificar los componentes en el terreno y su correlación con la historia estructural del montículo.

Este trabajo constituye uno de los primeros aportes en una etapa de estudios intensivos y localizados, a nivel de sitio, que contribuyen a la comprensión de las poblaciones que habitaron el Chaco Húmedo prehispánico. Asimismo, sienta las bases para el reanálisis de otros sitios identificados en la región (previamente estudiados desde un marco regional). Indagar sobre aspectos paleoclimáticos, asociados a las ocupaciones de los sitios de la región, nos permitirá observar si existen correlaciones con cambios en las estrategias utilizadas en el uso de la fauna. De esta manera contribuir a los estudios sobre movilidad, territorialidad, redes de interacción, intensificación, complejidad, entre otros.

Por último, a partir de esta aproximación preliminar, se destaca la importancia del abordaje integrado del registro arqueológico chaqueño. De manera específica, tal como se evidencia en el Sector Ribereño Paraguay - Paraná, el análisis de variables estrictamente zooarqueológicas pueden producir una interpretación incompleta del sitio, como por ejemplo la variabilidad temporal en la estructura de los recursos. En este sentido esperamos que esta aproximación sirva para poner en consideración la importancia del análisis integrado de fuentes de distinta naturaleza para orientar las preguntas zooarqueológicas chaqueñas. 
Agradecimientos: A Eduardo Bolo Bolaño por poner a disposición su querido El Cachapé, su pasión por la arqueología, amistad y ser parte del equipo. A Mariano Santini, Alejandro Tobisch, Marcos Plischuk, Ana Paula Porterie y Belén Aguirre que colaboraron en las sucesivas excavaciones. A Susana Salceda y Horacio Calandra por su constante ayuda. A los evaluadores cuyos comentarios contribuyeron a mejorar el manuscrito. A los editores por la invitación a participar del taller y del presente dossier. Estas investigaciones son financiadas por la Universidad Nacional de La Plata y el Consejo Nacional de Investigaciones Científicas y Técnicas (Argentina) a través de proyectos acreditados (PIP N0111 y 11/N846, respectivamente).

\section{Bibliografía citada}

Andrews, A. P.

1990 Owls, caves and fossils. University of Chicago Press, Chicago.

Behrensmeyer, A. K.

1978 Taphonomic and ecologic information from bone weathering. Paleobiology 4: 150162.

Behrensmeyer, A. K., C. T. Stayton y R. E. Chapman

2003 Taphonomy and ecology of modern avifaunal remains from Amboseli Park, Kenya. Paleobiology 29(1): 52-70.

Binford, L. R.

1981 Bones: Ancient Men and Modern Myths. Academic Press, New York.

Binford, L. R.

1984 Faunal Remains from Klasies River Mouth. Academic Press, Orlando.

Blumenschine, R. J., C. W. Marean y S. D. Capaldo

1996 Blind test of inter-analyst correspondence and accuracy in the identification of cut marks, percussion marks, and carnivore tooth marks on bone surfaces. Journal of Archaeological Science 23: 493-507.

Braunstein, J. A., S. A. Salceda, H. A. Calandra, M. G: Méndezy S. O. Ferrarini 2002 Historia de los chaqueños -Buscando en la "papelera de reciclaje" de la antropología sudamericana". Acta Americana, Journal of de Swedish Americanist Society 10 (1): 59-88.

Brizuela, S.

2010 Los lagartos continentales fósiles de la Argentina (excepto Iguania). Tesis Doctoral inédita. Facultad de Ciencias Naturales y Museo, UNLP, La Plata. 
Cabrera, A. y A. Willink

1973 Biogeografía de América Latina. Serie Biología. Monografía 13. OEA.

Calandra, H. A. y S. A. Salceda, S.A.

2004 El territorio y sus ocupantes: ¿Qué, quienes cómo y cuándo? Folia Histórica del Nordeste 15: 107-128.

De Feo, C., H.A. Calandra., M. Santini., B. Aguirre., G. Lamenza., M.I. Lanciotti., L. Del Papa y A. Porterie.

2003 Localización espacial y caracterización cultural de sitios arqueológicos del Gran Chaco Meridional. En: XXII Encuentro de Geohistoria Regional, Meichtry, N. (ed.), pp. 263-294. IIGHI - CONICET.

Echegoy, C.

1994 Arqueología del Paraná. Los fechados C14 de Arroyo Aguilar. Informe inédito. Museo Municipal de Arqueología y Paleontología de Reconquista. Reconquista, Santa Fe.

Ginzburg, R y J. Adámoli

2006 Situación Ambiental en el Chaco Húmedo. En La Situación Ambiental Argentina, A. Brown, M. Martínez Ortiz, M.Acerbi y J. Corcuera (eds.), pp. 103-113. Fundación Vida Silvestre Argentina, Buenos Aires.

Grayson, D. K.

1984 Quantitative Zooarchaeology: Topics in the analysis of Archaeological Faunas. Academic Press, Orlando.

Grayson, D. K.

1991 Alpine faunas from the White mountains, California: Adaptive Change in the Late Prehistoric Great Basin?.Journal of Archaeological Science 18: 483-506.

Grayson, D. K. y F. Delpech

1993 Changing Diet Breath in the Early Upper Paleolithic of Southwestern France. Journal of Archaeological Science 25: 1119-1129.

Grayson, D.K., F. Delpech, J. P. Rigaud y J. F. Simek

2001 Explaining the Development of Dietary Dominance by a Single Ungulate Taxon at Grotte XVI, Dordogne, France. Journal of Archaeological Science 28: 115-125.

Iriondo, $\mathrm{M}$.

2006 Cambios ambientales en el Chaco argentino y boliviano en los últimos miles de años. Folia Histórica del Nordeste 16: 39-50. 
Lamenza, G.

2013 El hombre y el ambiente en el Holoceno Tardío del Chaco Meridional. Universidad Nacional de Catamarca. Facultad de Ciencias Exactas y Naturales. San Fernando del Valle de Catamarca.

Lamenza, G.

2015a SIG y teledetección en investigaciones arqueológicas del Chaco argentino. Arqueología Iberoamericana 27: 40-54.

Lamenza, G.

2015b Utilización del análisis multivariante para la sistematización del componente alfarero del Chaco prehispánico. Arqueología Iberoamericana 28: 52-61.

Lamenza, G., M.Santini, H. Calandra y S. Salceda

2015 El Chaco Argentino: registro arqueológico regional y procesos de interacción. En ElCorazón de América del Sur 3. Arqueología de las Tierras Bajas de Bolivia y Zonas Limítrofes, S. Alconini y C. Jaimes Betancourt (eds.), pp. 155-173. Biblioteca del Museo de Historia, Universidad Autónoma Gabriel René Moreno, Santa Cruz de la Sierra.

Lamenza, G., S. Salceda y H. Calandra

2018a Identificación de componentes arqueológicos a través de técnicas numéricas: un caso de aplicación. Arqueología Iberoamericana S3: 35-37. http://purl.org/aia/S303.

Lamenza, G., H. Calandra y S. Salceda

2018b La Entidad Arqueológica Goya-Malabrigo y El Gran Chaco Sudamericano. En Goya-Malabrigo. Arqueología de una sociedad indígena del noreste argentino, G.Politis y M. Bonomo (eds.), pp. 307-332. UNICEN. Tandil.

Lyman, R. L.

1994 Vertebrate Taphonomy. Cambridge University Press, Cambridge.

Lyman, R. L. y K. M. Ames

2007 On the use of species-area curves to detect the effects of sample size. Journal of Archaeological Science 34: 1985-1990.

Meggers, B.

1994 Archeological evidence for the impact of mega-Niño events on Amazonia during the past two millennia. Climatic Change 28(4):321-338.

Mengoni Goñalons, G.

1988 Análisis de materiales faunísticos de sitios arqueológicos. Xama 1: 71-120.

Mengoni Goñalons, G. 
1999 Cazadores de guanaco de la estepa patagónica. Sociedad Argentina de Antropología, Buenos Aires.

Möhs, C. F.

1825 Treatise on mineralogy, or the natural history of the mineral kingdom. Archibald Constable, Edinburgo.

Morello, J. H. y J. Adámoli

1968 Las Grandes Unidades de Vegetación y Ambiente del Chaco Argentino. Primera parte. INTA, Serie Fitogeografía 10: 1-125.

Naumann, $\mathrm{M}$.

2006 Atlas del Gran Chaco Sudamericano. Sociedad Alemana de Cooperación Técnica (GZT). ErreGé\&Asoc., Buenos Aires.

Neiff, J. J., M. H. Iriondo y R. Carignan

1994 Large Tropical South American Wetlands: An Overview. En Proceedings of the International Workshop on the Ecology and Management of Aquatic-Terrestrial Ecotones, G. L. Link y R.J. Naiman (eds.),pp. 156-165. University of Washington, Washington.

Payne, S.

1975 Partial recovery and sample bias. En Archaeozoological Studies, A. T. Clason (ed.), pp. 7-17. North Holland, Amsterdam.

Popolizio, E.

2006 Contribución de la geomorfología a los estudios de antropología en el NEA. Folia Histórica del Nordeste 16: 75-82.

Porini, G.M.

2006 Proyecto Tupinambis. Una propuesta para el manejo de Tupinambis rufescens y T. merianae en la Argentina. En Manejo de Fauna Silvestre en la Argentina. Programas de Uso Sustentable, M. L. Bolkovic y D. Ramadori (eds.), pp. 65-75. Dirección de Fauna Silvestre, Secretaría de Ambiente y Desarrollo Sustentable, Buenos Aires.

Reitz, E. J. y E. S. Wing

1999 Zooarchaeology. Cambridge University Press, Cambridge.

Santini, M.

2009 Prehistoria de la región meridional del Gran Chaco: aportes del análisis de restos faunísticos en la reconstrucción de las estrategias adaptativas de los grupos aborígenes. Tesis Doctoral Inédita. Facultad de Ciencias Naturales y Museo, UNLP, La Plata. 
Scheinsohn, V.

1997 Explotación de materias primas óseas en la Isla Grande de Tierra del Fuego. Tesis Doctoral inédita. Universidad de Buenos Aires, Buenos Aires.

Shipman, P., G. F. Foster y M. Schoeninger

1984 Burnt bones and teeth: an experimental study of colour, morphology, crystal structure and shrinkage. Journal of Archaeological Science 11: 307-325.

White, T. E.

1953 A method of calculating the dietary percentage of various foods animals utilized by various aboriginal peoples. American Antiquity 18 (4): 396-398. 